Fixed Point Theory, 23(2022), No. 1, 371-384

DOI: $10.24193 /$ fpt-ro.2022.1.23

http://www.math.ubbcluj.ro/ nodeacj/sfptcj.html

\title{
GENERALIZED QUASI-CONTRACTIONS ON WEAK ORTHOGONAL METRIC SPACES
}

\author{
TANUSRI SENAPATI* ${ }^{*}$ ANKUSH CHANDA** AND VLADIMIR RAKOČEVIĆ ${ }^{* * *}$ \\ *Department of Mathematics, Gushkara Mahavidyalaya, West Bengal, India \\ E-mail: senapati.tanusri@gmail.com \\ **Department of Mathematics, National Institute of Technology Durgapur, India \\ and \\ Department of Mathematics, Vellore Institute of Technology, Vellore, India \\ E-mail: ankushchanda8@gmail.com \\ *** Department of Mathematics, Faculty of Sciences and Mathematics, \\ University of Niš, Niš, Serbia \\ E-mail:vrakoc@sbb.rs
}

\begin{abstract}
In this sequel, we introduce and study the concept of the weak orthogonal metric spaces as a generalization of the orthogonal metric spaces. Besides, we define and study the generalized quasi-contractions on such spaces and illustrate several non-trivial examples to endorse our obtained results. Among other things, as corollaries we obtain the main results of some of the pioneering articles existing in the literature. Finally, we answer the open question posed by Gordji et al. [On orthogonal sets and Banach fixed point theorem, Fixed Point Theory, 18(2):569-578, 2017].

Key Words and Phrases: Weak orthogonal relation, orthogonal metric space, orbital $O_{w^{-}}$ continuity, Banach $\perp$-contraction, fixed point.

2020 Mathematics Subject Classification: 47H10, 54H 25.
\end{abstract}

\section{ACKNOWLEDGEMENT}

The authors would like to thank the anonymous referee for his/her careful reading of the manuscript and useful comments which have undoubtedly improved the first version of the article.

\section{REFERENCES}

[1] R.P. Agarwal, E. Karapınar, D. O'Regan, A.F. Roldan-Lopez-de-Hierro, Fixed Point Theory in Metric Type Spaces, Springer, Switzerland, 2016.

[2] H. Baghani, M.E. Gordji, M. Ramezani, Orthogonal sets: The axiom of choice and proof of a fixed point theorem, J. Fixed Point Theory Appl., 18(2016), no. 3, 465-477.

[3] S. Banach, Sur les opérations dans les ensembles abstraits et leur application aux équations intégrales, Fund. Math., 3(1922), 133-181.

[4] V. Berinde, Iterative Approximation of Fixed Points, Springer, Berlin, 2007.

[5] S.K. Chatterjea, Fixed-point theorems, C.R. Acad. Bulgare Sci., 25(1972), 727-730. 
[6] Lj.B. Ćirić, A generalization of Banach's contraction principle, Proc. Amer. Math. Soc., 45(1974), no. 2, 267-273.

[7] Lj.B. Ćirić, On some maps with a nonunique fixed point, Publ. Inst. Math., 17(1974), 52-58.

[8] Lj.B. Ćirić, Some recent results in metrical fixed point theory, University of Belgrade, Beograd, 2003.

[9] M.E. Gordji, M. Rameani, M. De La Sen, Y.J. Cho, On orthogonal sets and Banach fixed point theorem, Fixed Point Theory, 18(2017), no. 2, 569-578.

[10] R. Kannan, Some results on fixed points, Bull. Calcutta Math. Soc., 60(1968), 71-76.

[11] W. Kirk, N. Shahzad, Fixed Point Theory in Distance Spaces, Springer, Switzerland, 2014.

[12] P. Kumam, N.V. Dung, K. Sitthithakerngkiet, A generalization of Ćirić fixed point theorems, Filomat, 29(2015), no. 7, 1549-1556.

[13] E. Malkowsky, V. Rakočević, Advanced Functional Analysis, CRC Press, Boca Raton, FL, 2019.

[14] M. Păcurar, I.A. Rus, Some remarks on the notations and terminology in the ordered set theory, Creat. Math. Inform., 27(2018), no. 2, 191-195.

[15] D. O'Regan, A. Petruşel, Fixed point theorems for generalized contractions in ordered metric spaces, J. Math. Anal. Appl., 341(2008), no. 2, 1241-1252.

[16] A. Petruşel, I.A. Rus, Fixed point theory in terms of a metric and of an order relation, Fixed Point Theory, 20(2019), no. 2, 601-622.

[17] M. Ramezani, Orthogonal metric space and convex contractions, Int. J. Nonlinear Anal. Appl., 6(2015), no. 2, 127-132.

[18] M. Ramezani, H. Baghani, Contractive gauge functions in strongly orthogonal metric spaces, Int. J. Nonlinear Anal. Appl., 8(2017), no. 2, 23-28.

[19] B.E. Rhoades, A comparison of various definitions of contractive mappings, Trans. Amer. Math. Soc., 226(1977), 257-290.

[20] I.A. Rus, Generalized Contractions and Applications, Cluj University Press, Cluj-Napoca, 2001.

[21] I.A. Rus, A. Petruşel, G. Petruşel, Fixed Point Theory, Cluj University Press, Cluj-Napoca, 2008.

[22] T. Senapati, L.K. Dey, B. Damjanović, A. Chanda, New fixed point results in orthogonal metric spaces with an application, Kragujevac J. Math., 42(2018), no. 4, 505-516.

[23] M. Turinici, Fixed point results on abstract ordered sets, Matematiche (Catania), 49(1994), no. $1,25-34$.

[24] M. Turinici, Contraction maps in ordered metrical structures, In: Mathematics Without Boundaries (P. Pardalos, T. Rassias - Eds.), Springer, New York, (2014), 533-575.

[25] W. Walter, Remarks on a paper by F. Browder about contraction, Nonlinear Anal., 5(1981), no. 1, 21-25.

Received: March 27, 2020; Accepted: November 16, 2020. 